\title{
Clinical Profile of Orbital Fractures with Special Reference to Blow Out Fractures
}

\author{
Liby Joseph ${ }^{1}$, Elizabeth John M. ${ }^{2}$ \\ ${ }^{1}$ Associate Professor, Department of Ophthalmology, Government Medical College, Kottayam, Kerala, India. \\ ${ }^{2}$ Professor and HOD, Department of Ophthalmology, Government Medical College, Kottayam, Kerala, India.
}

\section{ABSTRACT}

\section{BACKGROUND}

Orbital fractures usually occur as a part of polytrauma involving the midface. They range in severity from small minimally displaced fracture of an isolated wall that requires only conservative management, to major disruptions of orbit which require surgical intervention. Although several autogenous and alloplastic materials are available, autogenous bone grafting seems to give the best result. For larger defects, bone or titanium must be used for proper restoration of orbital anatomy. Fractures may involve the wall, rim or apex of the orbit, in isolation or in any combination and severity, and is usually associated with injuries to the eyeball and the peri orbital soft tissues.

\section{METHODS}

This is a descriptive study of 50 cases of orbital fractures that presented to the Department of Ophthalmology and Department of Maxillofacial Surgery at Govt. Medical College, Kottayam. All cases were analysed and categorized into different types of orbital fractures. The best mode and timing of treatment were decided in consultation with the Dept., of OMFS. Associated ocular injury was also assessed. Significant ocular injury was attended to as a priority.

\section{RESULTS}

$70 \%$ cases of orbital fractures were in the age group of 21 to 40 years of age of which $84 \%$ were males. The reason for male preponderance could be because they are more involved in outdoor activities and hence more susceptible to injury. $86 \%$ of cases were due to Road Traffic Accidents. Zygomatic bone was involved in 52\% cases. Blow out fractures of floor was $16 \%$ as compared to medial wall which was only $2 \%$. Most common indication for surgery was unacceptable facial deformity ( $52.17 \%$ ) followed by enophthalmos of more than $2 \mathrm{~mm}(26.08 \%)$.

\section{CONCLUSIONS}

In most cases of orbital fractures, eye escapes serious injury. Majority were treated conservatively. Among cases that underwent surgical intervention, early surgery within $2 \mathrm{wks}$. had the best postoperative results.

\section{KEY WORDS}

Orbital Trauma, Orbital Fractures, Blow Out Fracture, Enophthalmos, Diplopia
Corresponding Author:

Dr. Liby Joseph,

Associate Professor,

Department of Ophthalmology,

Government Medical College,

Kottayam, Kerala, India.

E-mail: libydoctor@gmail.com

DOI: $10.14260 / \mathrm{jemds} / 2019 / 683$

Financial or Other Competing Interests: None.

How to Cite This Article:

Joseph L, John EM. A clinical profile of orbital fractures with special reference to blow out fractures. J. Evolution Med. Dent. Sci. 2019;8(42):3151-3154, DOI: $10.14260 / \mathrm{jemds} / 2019 / 683$

Submission 30-05-2019, Peer Review 29-09-2019,

Acceptance 08-10-2019,

Published 21-10-2019. 


\section{BACKGROUND}

In this era of "speed" where speed thrills but kills, injuries to the orbit are seen in a significant number of persons involved in road traffic accidents. Today a head on collision in an automobile accident is the most common cause of orbital fracture. Indeed some $50 \%$ of orbital fractures may arise in this way. The orbit occupies a key anatomic position in the transitional zone between the midface and cranium, making it prone to injuries that are either isolated to the orbit or part of complex craniofacial fracture. Apart from this, the bony orbit is composed of comparatively thin bones, particularly in the inferomedial aspect. The complications of orbital fractures are primarily ophthalmic in nature and include aesthetic deformities such as enophthalmos, hypohthalmos as well as functional deficits such as diplopia and loss of vision.(1) Thus, ophthalmologists play a key role in the evaluation and management of orbital fractures. Early intervention in cases requiring repair, offers the best opportunity for improved aesthetic and functional results. Most orbital fracture repairs are performed under general anaesthesia. Orbital fractures are usually repaired in conjunction with other associated fractures of the craniofacial skeleton.(2) Surgery can safely be delayed for up to 14 days without producing any scarring or fibrosis.

\section{Surgical Technique of Repair of Blow Out Fractures of Floor}

The inferior orbital rim can be approached via skin incision. Once the inferior orbital rim is reached, an incision is made in the periosteum \& it can be bluntly elevated with the periosteal elevator. Once all entrapped tissues are released and the fracture site has been completely isolated, a decision is made regarding the placement of an orbital implant. A thin absorbable implant (e.g. Gel film) may be placed over the small fracture site as an added precaution against recurrent entrapment. For larger defects, an autogenous graft or alloplastics implant is necessary. Methods to secure the implant include using glue, placing a 'tab' at the anterior edge or direct fixation to the rim with a suture, wire, screws, or mini plates. After the orbital implant is secured, the periosteum is closed, and the eyelid incision is closed usually in a single layer

\section{Post-Operative Complications}

1. Related to malposition of the orbital rims

i. Persistent step - off fracture

ii. Bony mal-union.

iii. Frank dislocation of a portion of the orbital rim in the early post-operative period.

2. Related to soft tissue disruption

i. Displacement of the lateral canthus (Inferior Dystopia)

ii. Displacement of the medial canthus

iii. Damage to the nasolacrimal drainage system

3. Related to vision: visual loss may be caused by intra operative manipulations of the globe or optic nerve or by post - operative orbital haemorrhage that produces central retinal artery occlusion or compressive optic neuropathy.

4. Complications related to repair of internal orbital wall fractures

i. Persistence of enophthalmos or hypohthalmos ii. Persistent ocular motility deficits \& diplopia

iii. Extrusion, migration and infection of alloplastic implants and bone grafts.

5. Globe malpositions.

The primary consideration in the management of orbital fractures is to determine which fractures require surgery and when such intervention should be undertaken. Surgery itself carries some risks including failure of the procedure to correct the deformity, the possibility of worsening diplopia, the rare risk of visual loss and systemic complications of general anaesthesia. Therefore, in the group of patients with relatively less severe orbital fractures, the risk benefit ratio of surgery must be weighed against the corresponding riskbenefit ratio of more conservative treatment.

\section{METHODS}

\section{Study Design}

Descriptive study.

\section{Duration}

12 months.

\section{Study Population}

Patients attending Ophthalmology OP and Oral and Maxillofacial Department with orbital fractures.

\section{Sample Size}

The patients were selected by convenient sampling technique. All patients coming to the institute with orbital fractures were included in the study. The sample size has been approved by the Institutional review Board and the Scientific Research committee.

\section{Study Procedure}

After obtaining the IEC/IRB clearance (27/11), the study was commenced by including all patients who attended the casualty department of Ophthalmology and that of the Oral and Maxillofacial surgery casualty with orbital fractures during the study period and satisfying the inclusion and exclusion criteria. Informed consent was obtained from all the participants after explaining to them the purpose of the study. Initial evaluation was done to determine the extent of associated systemic and ocular injuries. The highest preference was given to life threatening injuries which were managed as a priority. A complete history was obtained including mechanism of injury as well as whether there was any loss of consciousness. Assessment of ocular function inthe emergency setting included examination of the anterior segment especially for the position of eyeball, pupillary reaction, visual acuity, ocular movements and the intactness of orbital margins. The face was inspected and palpated for possible associated facial and nasal deformities, crepitus, areas of anaesthesia or hypoaesthesia. Slit lamp examination and dilated fundus examination were performed to assess the degree of injury to the globe. Forced duction and forced generation tests were done when orbital oedema had largely subsided. Enophthalmos if present, was measured with exophthalmometer. Cases with rupture globe were repaired on the same day. Radiological examination with plain $\mathrm{X}$ ray 
and CT scan was done in all cases. Consultations with maxillofacial surgery and ENT departments were done in all cases. Neurosurgery consultation was done in required cases. Patients were evaluated on the first, third, fifth, and seventh days and after two weeks to assess the extent of enophthalmos, recovery of ocular movement, diplopia and facial deformity. All patients were thoroughly assessed, and decision was taken regarding the mode of management in concurrence with the Oral and maxilla facial department. Those who needed surgical intervention, surgery was done in the department of maxillofacial surgery.

Surgical intervention was done when at least any one of the following indications were present

1. Cosmetically unacceptable facial deformity

2. Enophthalmos of more than $2 \mathrm{~mm}$

3. Functionally debilitating diplopia in primary gaze position

4. Clinical and CT evidence of frank muscle entrapment in blow out fractures

5. Displaced fractures especially involving the orbital rim.

Conservative management was done in cases with the following indications

1. Cosmetically acceptable facial deformity

2. Undisplaced fractures

3. Minimal restriction of ocular movements with diplopia in extreme gaze only

4. No clinical or CT evidence of muscle entrapment with negative forced duction test.

Cases with traumatic optic neuropathy were treated with 'megadose' I/V methyl prednisolone for 3 days followed by rapid tapering of oral prednisolone for twelve days. All patients, both surgically treated and conservatively treated were evaluated after two weeks, 1 month, two months and six months interval. Parameters mainly evaluated were correction of facial deformity and enophthalmos, recovery of ocular movements and diplopia.

\section{Statistical Analysis}

The data was entered in Microsoft Excel and analysed using SPSS version 16. Continuous variables were expressed in mean and standard deviation. Types of fractures, Clinical features, mode of injury, mode of treatment, pre and postoperative findings were analysed. The above categorical variables were expressed in proportions.

\section{RESULTS}

\begin{tabular}{|c|c|c|}
\hline Mode & No. of Cases & Percentage \\
\hline Road traffic accidents & 23 & 46 \\
\hline Fall & 15 & 30 \\
\hline Assault & 9 & 18 \\
\hline Accidental blunt trauma to orbit by a moving object & 3 & 6 \\
\hline \multicolumn{2}{|c|}{ Mode of Injury } \\
\hline
\end{tabular}

$70 \%$ cases of orbital fractures were in the age group of 21 to 40 years. $84 \%$ of cases were males and $16 \%$ cases were females.

Ocular injury was seen in $42 \%$ of cases. Rupture globe was seen in 2 cases which was repaired first before attending to the orbital fracture. Lid laceration was seen in one case of fracture of infra orbital rim and zygomatic complex. Traumatic optic neuropathy was seen in 8 cases which were treated with Mega dose of Intravenous Methyl Prednisolone. 2 cases had no perception of light. Other ocular injuries seen were hyphaema, Iridodialysis, Traumatic mydriasis, Subluxation of lens, Vitreous haemorrhage and Commotio retinae.

There was no perception of light in 2 cases of traumatic optic neuropathy and 1 case of rupture globe. There was just perception of light in another case of rupture globe. There were 4 cases with visual acuity between counting fingers close to face and 5/60. Of these, 3 cases were of traumatic optic neuropathy.

There were 6 cases with orbital roof fractures. Mid facial fractures including Le Fort II, Nasomaxillary, and malar fractures were seen in 23 cases. There was one case with fracture of medial wall of orbit without blow out. 7 cases had fracture of Lateral Orbital margin and zygoma complex. Isolated superior or inferior orbital margin was seen in 6 cases.

All cases showed marked lid oedema. Enophthalmos was seen in 11 cases out of the 50 cases included in the study. 6 cases had enophthalmos of more than $2 \mathrm{~mm}$. One case that presented after 3 months of injury had $5 \mathrm{~mm}$ of enophthalmos. Proptosis was seen in one case due to retrobulbar haemorrhage. Diplopia was seen in 6 cases. Out of these, 4 had functionally debilitating diplopia in primary gaze. Facial deformity due to depression of malar eminence and irregularity of orbital margins was seen in 17 cases. Hypoaesthesia along the distribution of infra orbital nerve was seen in 8 cases.

Out of the total 50 cases, 27 cases were treated conservatively, and 23 cases were treated surgically. Among the cases that underwent surgical intervention, the repair was done within 2 weeks of injury. Pre-operative clinical features in the surgically treated group were unacceptable facial deformity in 12 cases, enophthalmos in 6 cases, diplopia in primary position in 3 cases, hypotropia and ptosis in one case each.

Enophthalmos, diplopia persisted in one case each, cosmetic deformity in 2 cases and infra orbital hypaesthesia in 3 cases. The enophthalmos persisted but to a lesser degree in one case of blow out fracture that had presented after 6 weeks of injury. During surgery also, it was very difficult to release the incarcerated muscle which showed obvious fibrotic changes. Diplopia also persisted in the same case.

11 cases had restriction of ocular movement with no diplopia and negative forced duction test. 5 cases each had acceptable facial deformity and enophthalmos less than 2 $\mathrm{mm}$. 3 cases had infra orbital hypaesthesia.

Enophthalmos persisted in 5 cases, acceptable facial deformity in 3 cases, restricted ocular movements with diplopia in 2 cases and hypaesthesia in 1 case. Enophthalmos persisted in all the cases treated conservatively.

There were 9 blowout fractures in the 50 cases included in the study. 8 were blow out fractures of floor and 1 was of the medial wall. Out of the fracture floor of the orbit, six were pure and two were impure fractures. Blow out fractures were treated surgically in 5 cases (56\%) and conservatively in 4 cases $(44 \%)$. 
Silastic sheet was used in 2 cases (40\%) and anterior wall of maxilla was used in 3 cases $(60 \%)$

\section{DISCUSSION}

Orbital fractures were found to occur most commonly in young adults in the age group of 21-30 years (40\%). In a study by Motamedi. et al (3), the incidence was $59 \%$ in the 20 29 years age group. In the present study of orbital fractures, the youngest patient was 6 years old (Road Traffic Accident) and the oldest was 75 years old (Road Traffic Accident).

Males accounted for the vast majority of cases (84\%). The reason for the male preponderance in all the studies is probably because males are more involved in outdoor activities and hence are more susceptible to injury.

In most cases of orbital fractures, eye escapes serious injury. Associated significant ocular injury was present in $42 \%$ cases in this study which included rupture globe, vitreous haemorrhage and traumatic optic neuropathy. Apex, lateral wall and Le Fort III fractures had greater association with serious ocular injuries. Cook et al (4) has reported an incidence of $26 \%$ of associated ocular injuries in orbital fractures.

There was no perception of light in 3 cases in this study. Two were cases of traumatic optic neuropathy in frontal bone and roof fractures and one was a case of extensive rupture globe with collapse of the eyeball. Majority had good visual acuity. $56 \%$ had $6 / 9$ or better. The incidence of traumatic optic neuropathy in this study was $16 \%$ while in another study conducted by Amrith et al(5) it was $20 \%$.

In the present study, zygomatic complex was the most frequently involved bone (44\%) followed by maxilla (16\%). Brand et $\mathrm{al},(6)$ in his study has reported the incidence of zygomatic fractures as $57 \%$.

The incidence of blow out fractures was 18\%. 16\% had blow out floor and $2 \%$ had blow out fractures of medial wall.

In this study, $46 \%$ of the total cases of orbital fractures underwent surgical intervention. In blow out fractures, surgical repair was done in $55.55 \%$ cases.

The most common indication for surgery was unacceptable facial deformity $(52.17 \%)$ followed by enophthalmos of more than $2 \mathrm{~mm}$

Among the cases that underwent surgical intervention $86.95 \%$ were repaired within the first two weeks of injury and the post-operative results were excellent as compared to those done later.

Following surgery, enophthalmos was corrected in $83.4 \%$ cases and functionally debilitating diplopia was relieved in $67 \%$ of cases.

For the surgical repair of blow out fractures of the floor of the orbit, anterior wall of maxilla was used in $60 \%$ of cases and silastic sheet in $40 \%$ cases. Both these did not show any post-operative complications, and both had equally good recovery of function and cosmesis.

Of the 54\% cases managed conservatively, enophthalmos persisted in all the cases and diplopia persisted in $66.66 \%$ cases. Acceptable facial deformity persisted in $60 \%$ cases. Full range of ocular movement was regained in $81.2 \%$ cases.

\section{CONCLUSIONS}

Orbital fractures were more common between 21-40 years $(70 \%)$. Majority of cases were males (84\%). The most common mode of injury leading to orbital fractures was Road Traffic Accidents (46\%). The most common bone involved in orbital fractures was zygomatic complex (52\%). Majority of cases did not have serious eye injury. Blow-out fractures of floor were more common (16\%) than medial wall (2\%). Pure blow out floor fractures were more common than impure blow out. Orbital floor reconstruction required either an autologous bone or a synthetic material as implant. Majority of the cases were treated conservatively. Early operation (within two weeks of injury) was easier with better results and fewer complications. Enophthalmos, diplopia and facial deformity were relieved in majority of cases treated surgically while it persisted in majority of cases that underwent conservative management. A multi-team approach involving the ophthalmologist and the maxillofacial surgeon is important for restoring maximal function and cosmesis in orbital fractures.

\section{REFERENCES}

[1] Aladelusi T, Akinmoladun V, Olusanya A, et al. Analysis of Road Traffic Crashes-Related Maxillofacial Injuries Severity and Concomitant Injuries in 201 Patients Seen at the UCH, Ibadan. Craniomaxillofac Trauma Reconstruction 2014;7(4):284-9.

[2] Harstein ME, Roper-Hall G. Update on orbital floor fractures: indications and timing for repair. Facial and Plastic Surg 2000;16(2):95-106.

[3] Motamedi MHK. An assessment of maxillofacial fractures: a 5-year study of 237 patients. J Oral Maxillofac Surg 2003;61(1):61-4.

[4] Cook T. Ocular and periocular injuries from orbital fractures. J Am Coll Surg 2002;195(6):831-4.

[5] Amrith S, Saw SM, Lim TC, et al. Ophthalmic involvement in craniofacial trauma. J Craniomaxillofacial Surg 2000;28(3):140-7.

[6] Brandt KE, Burruss GL, Hickerson WL, et al. Management of mid-face fractures with intracranial injury. J Trauma 1991;31(1):15-9. 